\title{
Soip over Satellite Testing - TIM Experience
}

\author{
Giulio Maggiore', Letterio Pirrone ${ }^{2}$, Gaethan Donlap Kouang ${ }^{2}$, \\ Federico Piovan ${ }^{3}$, and Biagio Ricco Galluzzo ${ }^{3}$ \\ ${ }^{1} \mathrm{TIM}$, \\ via del Giorgione 159, 00147 Roma \\ gmaggiore@mail.tim.it \\ ${ }^{2}$ EUTELSAT, \\ lpirrone@eutelsat.fr, gdonlap@eutelsat.fr \\ ${ }^{3}$ Telecom Italia Lab, \\ via Reiss Romoli 274, 10148 Torino, \\ federico.piovan@tilab.com, biagio.ricco@tilab.com
}

\begin{abstract}
In the present Mobile Telecommunications scenario, the transmission of SS7 signalling on traditional TDM circuits is evolving to a SS7 over IP network (SoIP) solution, due to the continuous efficiency research in optimizing transmission and to the continuous goal of cost reduction. Some operators are using SoIP network in a national environment, while international SoIP networks are going to be deployed in a few years. Since transmissions over satellite may represent a fast way to deploy an international Backbone, TIM performed a trial in cooperation with TILAB, using Cisco Signalling Gateways and EUTELSAT satellite network. This paper describes the most significant results obtained by this experience, with respect to two critical aspects of satellite connection: the Transmission Delay and the Bandwidth Availability. Satellite delay put in evidence some practical limitations for the current Cisco release (called MB9), if compared with the traditional terrestrial Backbone; a special upgraded release (MB9-SAT) resolved them, but dynamic allocation of bandwidth asked for a detailed investigation on configuration criteria, in order to avoid system congestion. Both releases have been tested also with a delay-line simulator, to examine their performances on traffic management with delay varying between 0 and $700 \mathrm{~ms}$. These activities drove us to outline an operative reference model, useful to understand network behavior under different conditions and to configure its fundamental parameters.
\end{abstract}

\section{Glossary}

$\begin{array}{ll}\mathrm{B}_{\mathrm{ETH}}: & \text { Signalling on Ethernet traffic Band } \\ \mathrm{B}_{\mathrm{IN}}: & \text { Traffic Band injected into IP Network } \\ \mathrm{B}_{\mathrm{IN}, \max }: & \text { Maximum threshold of } \mathrm{B}_{\mathrm{IN}} \text { before congestion } \\ \mathrm{B}_{\mathrm{S}}: & \text { Total Satellite Band } \\ \mathrm{B}_{\mathrm{SS} 7}: & \text { Signalling traffic Band } \\ \Delta \mathrm{B}: & \text { Increase of Signalling traffic band } \\ \mathrm{CWND}: & \text { Congestion Window } \\ \text { DAMA: } & \text { Dynamic Assignment Multiple Access }\end{array}$

R. Groz and R.M. Hierons (Eds.): TestCom 2004, LNCS 2978, pp. 112-127, 2004.

(C) IFIP 2004 


\begin{tabular}{|c|c|}
\hline DPC: & Destination Point Code \\
\hline EUTELSAT: & Satellite Communications company \\
\hline GTT: & Global Title Translation \\
\hline IETF: & Internet Engineering Task Force \\
\hline IP: & Internet Protocol \\
\hline M2PA: & MTP2 user Peer-to-peer Adaptation layer \\
\hline MSU : & Message Signalling Unit \\
\hline MTP2: & Message Transfer Part 2 \\
\hline MTP3: & Message Transfer Part 3 \\
\hline $\mathrm{N}_{\mathrm{A}}:$ & SCTP Associations' Number \\
\hline OSI: & Open System Interconnection \\
\hline PAMA: & Permanent Assignment Multiple Access \\
\hline RTT: & Round Trip Time \\
\hline SIGTRAN : & Signalling Transport \\
\hline SCCP: & Signlling Connection Control Part \\
\hline SCTP: & Stream Control Transmission Protocol \\
\hline SG: & Signaling Gateway \\
\hline SoIP : & Signalling SS7 over Internet Protocol \\
\hline SS7: & Signalling System n.7 \\
\hline $\mathrm{T}_{\mathrm{ACK}} / \mathrm{T}_{\mathrm{ALL}}:$ & ACKnowledge / ALLocation Time \\
\hline TCP: & Transport Control Protocol \\
\hline TDM: & Time Division Multiplexing \\
\hline TDMA: & Time Division Multiple Access \\
\hline TILAB: & Telecom Italia LABoratories \\
\hline TIM: & Telecom Italia Mobile S.p.A. \\
\hline TT: & Traffic Terminal \\
\hline VLAN: & Virtual Local Area Network \\
\hline WAN: & Wide Area Network \\
\hline
\end{tabular}

\section{Introduction}

Satellite communication represents the pioneering stage of telecommunication network in those parts of the world not covered by transmissions infrastructures: it allows a fast deployment of a telecommunication network, giving access to those services typically provided by the wire line and wireless terrestrial networks.

The paper presents some results of SoIP over satellite testing, implemented with CISCO Signalling Gateways, at first equipped with release MB9, then with a special release for "SoIP over satellite" called MB9-SAT. Both of them have been tested also with a delay-line simulator, in order to examine their performances under different delay conditions. Results and observations lead to outline an operative reference model, mainly aimed to guide in the configuration of fundamental transport parameters.

\section{Soip over Satellite Testing}

The acronym SoIP (Signalling SS7 over IP) can be referred to every system dealing with transport of signalling messages over an IP network. 
Signalling Gateways (SG) allow SS7 messages to be transported on IP networks, according to the architectural model proposed by SigTran (IETF Working Group). Usually they are composed of several modules, each of them performing the major functionalities, as shown in Figure 1:

- the line interfaces, that set up the signalling circuits front-end, realizing the interworking function of MTP2 layer messages;

- the central processor, that performs MTP3 and SCCP layers, managing the connection;

- the IP Fast Ethernet Interfaces, that perform system connections to IP backbone.

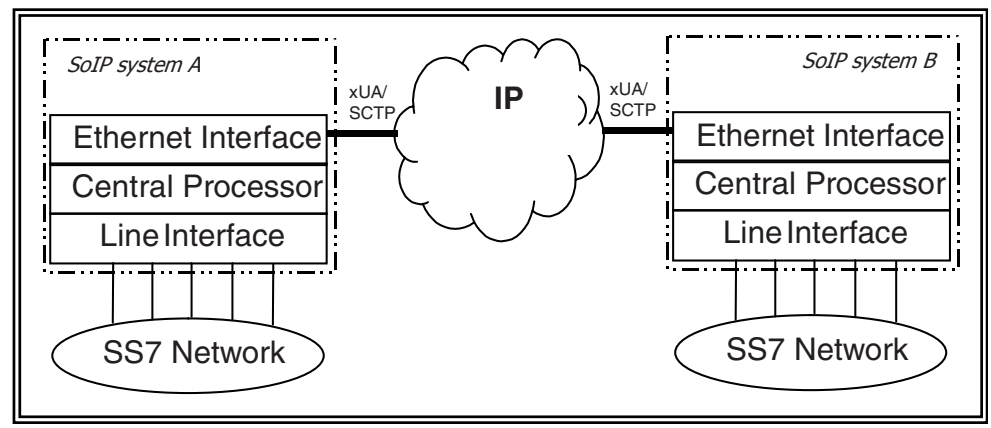

Fig. 1. SoIP System general architecture

Concerning with IP transport, the protocol stack defined by SigTran specifies the replacement of the TCP protocol (layer 4 of OSI reference model) with the new SCTP protocol [1]. The TCP is designed in order to ensure the transfer of long files without the control of the transit time; instead, the SCTP is designed in order to satisfy the timing requirements of the signalling by containing transit delays. Interworking functions between SS7 and IP protocols rely on an adaptation layer, positioned between transport and application layers, which performs adaptation at SS7 layer 2, or 3 and higher, according to manufacturer's strategies [2], [3], [4].

\subsection{Testing Architecture}

The system under test is composed by the following basic equipments (see Fig. 2):

- SS7 Traffic Simulator, used as load generator, to send and receive the signalling protocols messages. It is able to generate Message Signalling Unit (MSU) of different length (60, 120 and 270 Bytes length are commonly used) and with two types of SS7 routing (via DPC in MTP level or GTT in SCCP level). The Traffic Simulator has a 30 Signalling Links limit capacity, each link able to transport up to $64 \mathrm{~Kb} / \mathrm{s}$, so that 30 Erlang bi-directional is the maximum traffic load.

- Signalling Gateways (A and B) are Cisco ITP7500, transporting SS7 layer 3 (MTP3) on SCTP through the adaptation layer M2PA [5], while IP network layer is transferred through a Fast Ethernet. 
SG's performances on IP terrestrial backbone have already been estimated in latest trials, where each SG was able to get over 0.98 - 1 Erlang per link on 30 connected Signalling Link, varying MSU's length.

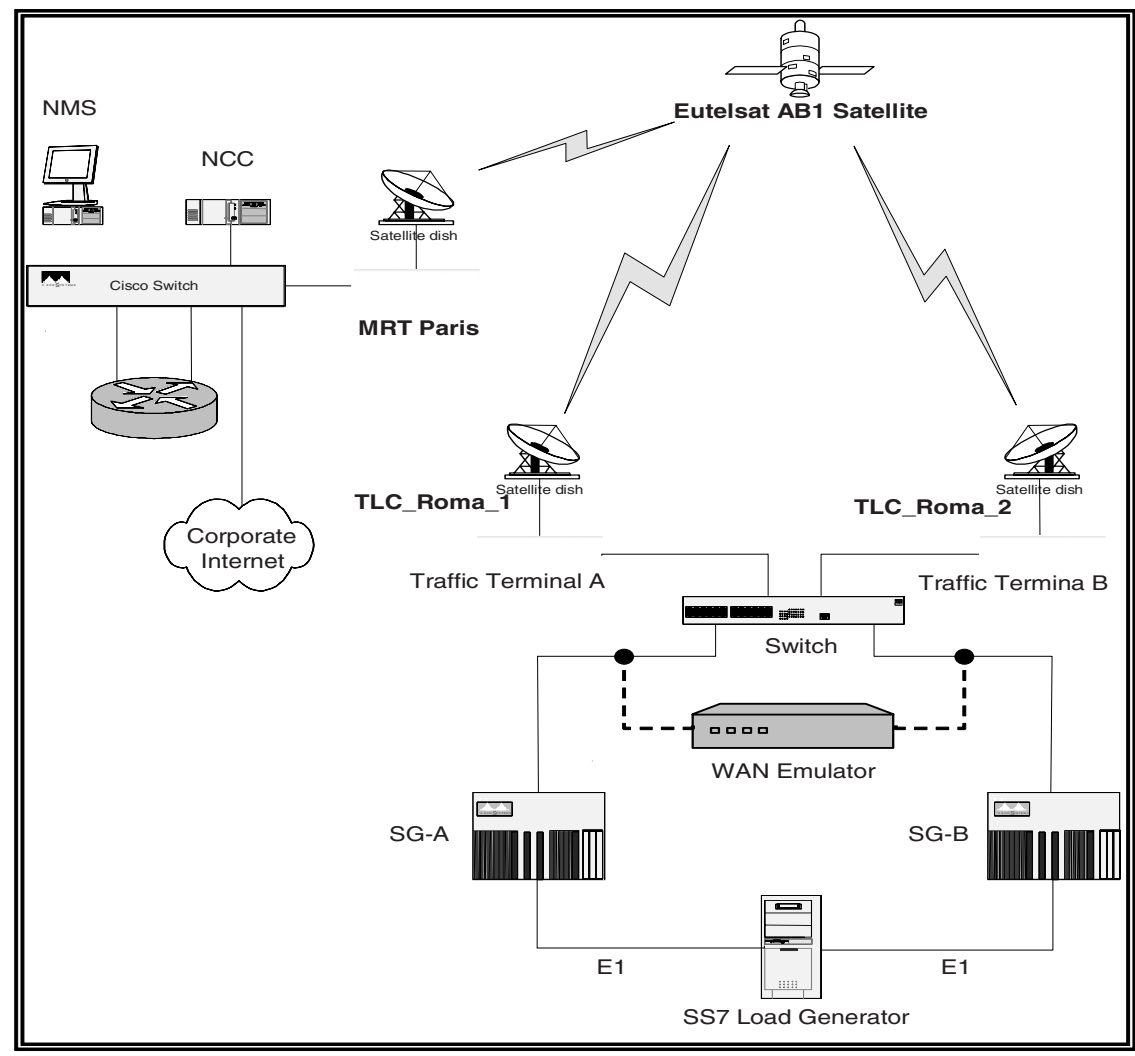

Fig. 2. SoIP over Satellite Testing Architecture

- Traffic Terminal: interface device between Ethernet and Satellite networks, described in paragraph 2.2.

- WAN Emulator: device emulating a Wide Area Network (WAN), whose basic features are fully configurable, such as network bandwidth, packet delay time, packet loss rate, packet's priority, etc. etc. Along the trial it was used only as a delay-line simulator, to allow a detailed performance measurement on delay variation.

\subsection{Satellite Connection}

EUTELSAT provides the space segment and the satellite connectivity by Linkway Platform from ViaSat Inc., a pay-per-use satellite communication system based on Traffic Terminals: these devices can transport multiple protocols traffic (IP, ATM, Frame Relay, ISDN and Signaling System 7) through a native 10BaseT Ethernet con- 
nection to the terrestrial networking equipment, manage the IP routing table and assign dynamical satellite bandwidth as needed.

Remote configuration and control of the entire network are carried out by the Network Control Computer (NCC), connected to the Master Reference Terminal (MRT), installed at EUTELSAT Headquarters in Paris, where the network administrator can configure all the parameters in real time: a complete network becomes a cost-effective solution, thanks to the efficient utilization of space segment resources by TDMA access and their sharing among all terminals by dynamic allocation of bandwidth.

As for the testing network (see Fig. 2), Signalling Gateways SG-A and SG-B communicate with Traffic Terminals TT1 and TT2 through a Layer-2 Switch, where two VLANs separate the traffic between SG-A and TT1 from the traffic between SGB and TT2: Linkway modems encapsulate the Ethernet traffic into the satellite access protocol and transmit bursts to each other on a Permanent Virtual Circuit, using TDMA access mode.

The requested satellite bandwidth is obtained by two carriers, both of them dynamically accessible from terminals, according to the Multi Frequency TDMA protocol (only one terminal transmitting on a carrier at the same time under control of the MRT); taking into account the protocol headers and the guard time between the TDMA bursts, the maximum available bandwidth per carrier is $2320 \mathrm{Kbps}$.

This value represents the maximum full duplex Ethernet throughput on satellite, available both as full Permanent Assignment Multiple Access (PAMA, i.e. 2048 $\mathrm{Kb} / \mathrm{s}$ ) Band and as mixed combination of Permanently Assignment (i.e. $1024 \mathrm{~Kb} / \mathrm{s}$ ) and Dynamic Assignment Multiple Access (DAMA, exceeding) Band.

\subsection{Test Typologies}

It's in common experience that IP transport is prone to delays, which can be emphasized by interworking functions between the different protocols in use and, in this case, by the satellite connection: that's why more attention is asked to parameters such as packet delay, loss rate [6] and satellite bandwidth availability in testing innovative signalling transport systems.

In fact, performance testing has the purpose to evaluate the traffic level (in terms of Loss Rate and Transit Delay) that can be handled respecting SS7 standard specifications, while varying MSU length, SS7 routing (via DPC in MTP level or GTT in SCCP level ) and the bandwidth allocation on the satellite network.

The trial's main target was to evaluate critical impacts of the satellite connection usage on the whole system, as introduced in the following paragraph.

\subsection{Critical Aspects}

The satellite connection introduces two critical aspects on the system: the Transmission Delay and the Bandwidth Availability.

A traditional SoIP system introduces a $20-40$ ms delay due to processing on single SGs, which complies with acknowledges timers of SS7 messages. Now, satellite connection introduces 640-680 ms Round Trip Time (RTT), that means the entire system's RTT will be about 700 ms: it's necessary to evaluate the impact of Transmis- 
sion Delay on the SS7 messages time-outs and on the SCTP retransmission timers [7], [8], [9].

Satellite bandwidth can be assigned in two different ways: PAMA Band is permanently assigned on satellite, whereas DAMA Band is assigned on demand and if available, according to a specific negotiating algorithm, with configurable allocation/de-allocation speeds. When using PAMA Band, the satellite connection affects entire system only by introducing its nominal delay; when using DAMA Band it's necessary to estimate the negotiating algorithm incidence on entire system delay and on possible SG congestions.

\section{Main Results}

\subsection{Transmit Delay Effects}

In order to investigate the transmit delay effects on satellite link (about $700 \mathrm{~ms}$ RTT), system equipments have been configured as follows:

- Satellite band: PAMA Band at $2048 \mathrm{~Kb} / \mathrm{s}$ with about $150 \mathrm{~Kb} / \mathrm{s}$ DAMA Band, so that there is no bottleneck on this side;

- Signalling Gateway: IOS in Release MB9 and one SCTP association, with Congestion Window (CWND) growing from initial value to 64000 Bytes (maximum value), as configured by manufacturer.

CWND is one of the SCTP variables regulating the transmission rate, as focused later on.

Two are the anomalous consequences we observe:

1) when generated traffic stays between 400 and $850 \mathrm{~Kb} / \mathrm{s}$ in Ethernet Band, there is a transient congestion up to $70 \mathrm{~s}$ (loss rate $40-70 \%)$;

2) when traffic exceeds $850 \mathrm{~Kb} / \mathrm{s}$, congestion lasts for more than $240 \mathrm{~s}$.

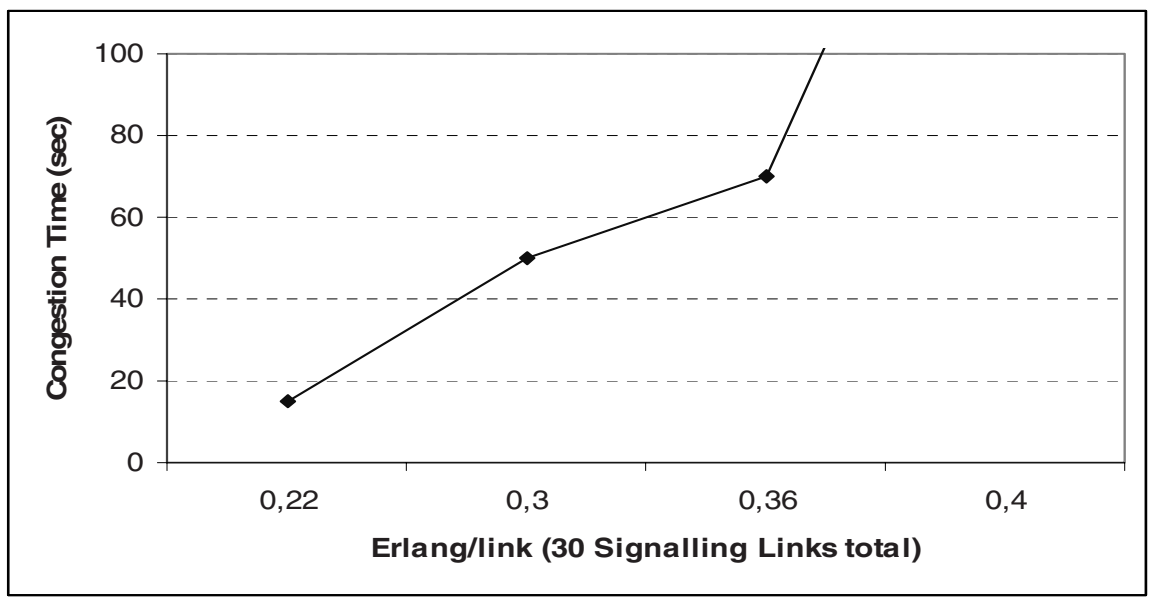

Fig. 3. Congestion Time vs. Erlang/link (Rel. MB9) 
Figure 3 shows how congestion duration increase with generated SS7 traffic: when load generator works at 0.36 Erlang/link, there is a transient congestion of about $70 \mathrm{~s}$ that limits Transmitted Band to $300 \mathrm{~Kb} / \mathrm{s}$, even if generated band is about $1020 \mathrm{~Kb} / \mathrm{s}$. This is connected with CWND rising from initial value to the needed level.

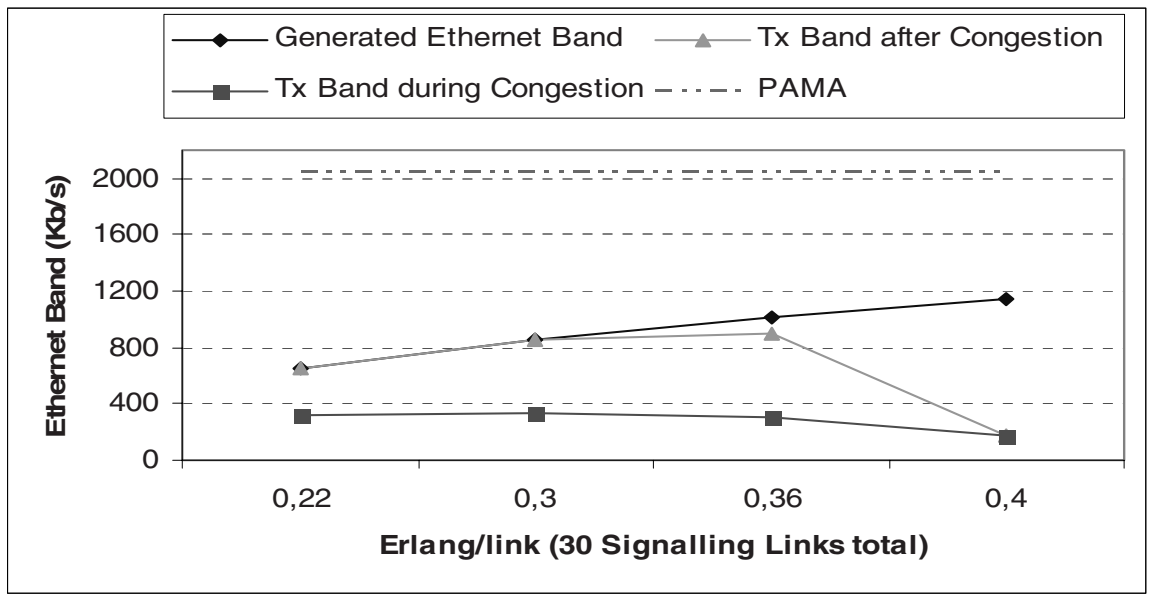

Fig. 4. Generated and Transmitted Ethernet Band vs. Erlang/link (Rel. MB9)

In Figure 4 there's an example of how Transmitted Band vary with generated SS7 traffic (both during and after congestion): when traffic is 0.36 Erlang/link, Transmitted Band is $300 \mathrm{~Kb} / \mathrm{s}$ during $70 \mathrm{~s}$ of transient congestion and rises to $900 \mathrm{~Kb} / \mathrm{s}$ after it, even if generated band is about $1020 \mathrm{~Kb} / \mathrm{s}$.

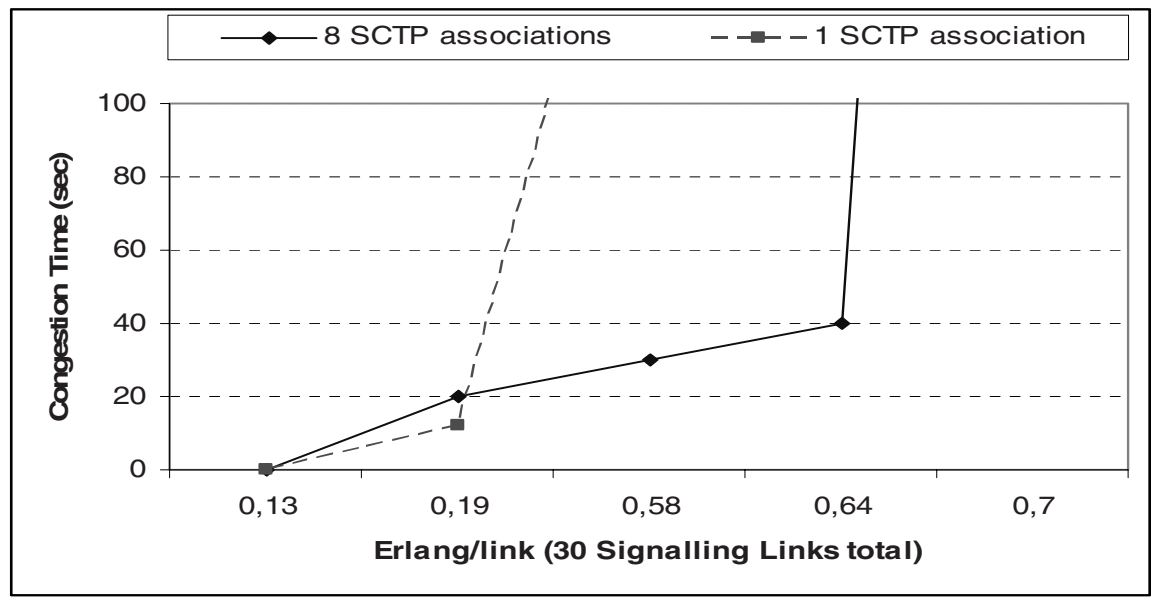

Fig. 5. Congestion Time vs. Erlang/link (8 associations, Rel. MB9) 
To improve such a limited performance, the simplest step is activating more than one SCTP associations, even if it implies that occupied Ethernet band increases, SS7 traffic being equal, according to number of activated and then used associations.

Moreover uniform distribution of SS7 traffic among all SCTP associations is not granted, because SG strictly divides messages according to their Signalling Link Code, when in-sequence delivery is required: thus, the SS7 interface configuration (link-sets amount and dimension) could influence packet distribution and congestion conditions as well.

Setting Association number $\mathrm{N}_{\mathrm{A}}=8$ (maximum link-sets dimension) obtains a little improvement on critical behaviour, without totally solving it (see Figures 5 and 6): the initial congestion is shorter in time and lower in percentage (duration 20-40 s, loss rate 5-20\%), and it becomes a continuous state after a higher threshold (while generated traffic doesn't exceed $1900 \mathrm{~Kb} / \mathrm{s}$, equal to $0.64 \mathrm{~Kb} / \mathrm{s}$ in this case), but it is still unacceptable, bringing forward that $\mathrm{N}_{\mathrm{A}}$ is not decisive.

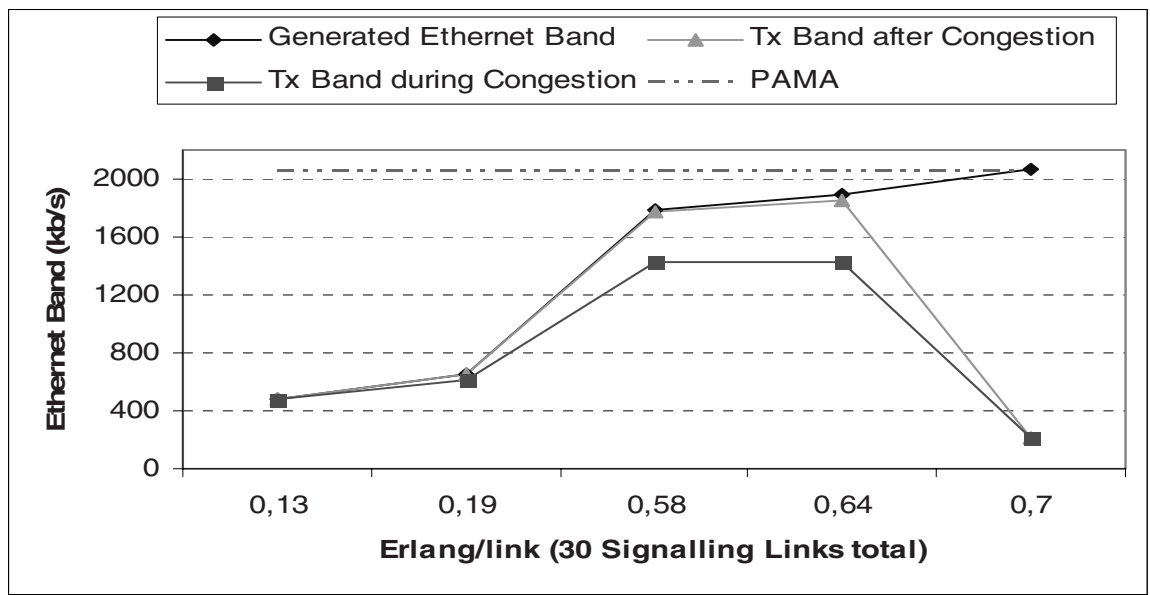

Fig. 6. Generated and Transmitted Ethernet Band vs. Erlang/link (8 associations, Rel. MB9)

For a further examination of the single SCTP association behavior varying RTT, we replace the satellite connection with a WAN Emulator and increase RTT value on this instrument: congestion happens when RTT exceeds $600 \mathrm{~ms}$, with loss rate growing with RTT itself, independently from MSU length (60 or 270 Bytes) and SS7 routing (DPC or GTT), as shown in Figure 7.

This result confirms RTT on satellite link is so high that the amount of data waiting for acknowledge (called OUTSTAND) reaches CWND Maximum Value too fast, driving the system to a congestion state: that's why the configuration of the single association shall be modified in terms of SCTP parameters.

In order to make them accessible to operator, manufacturer modifies SG Operative System (special Release MB9-SAT), suggesting values in brackets: Initial CWND Size (384000), Retransmit CWND Rate (0, SCTP Fast Restart), Fast CWND Rate (0), Transmission Queue Depth (8000); besides, CWND never grows over its initial value. 


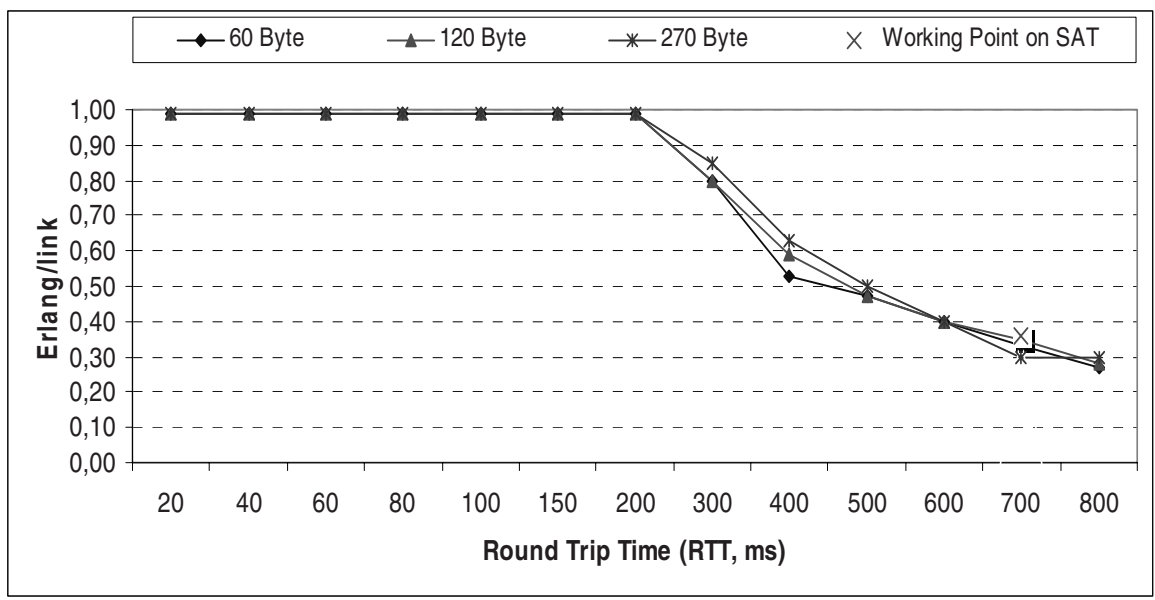

Fig. 7. Traffic Limitation vs. RTT (1 association, Rel. MB9)

Experimental checking with WAN Emulator shows complete absence of congestion through only one SCTP association, even when SS7 traffic rises suddenly to 30 Erlang (see Figure 8).

After this updating, we can proceed to evaluate bandwidth availability effects, coming back to satellite connection.

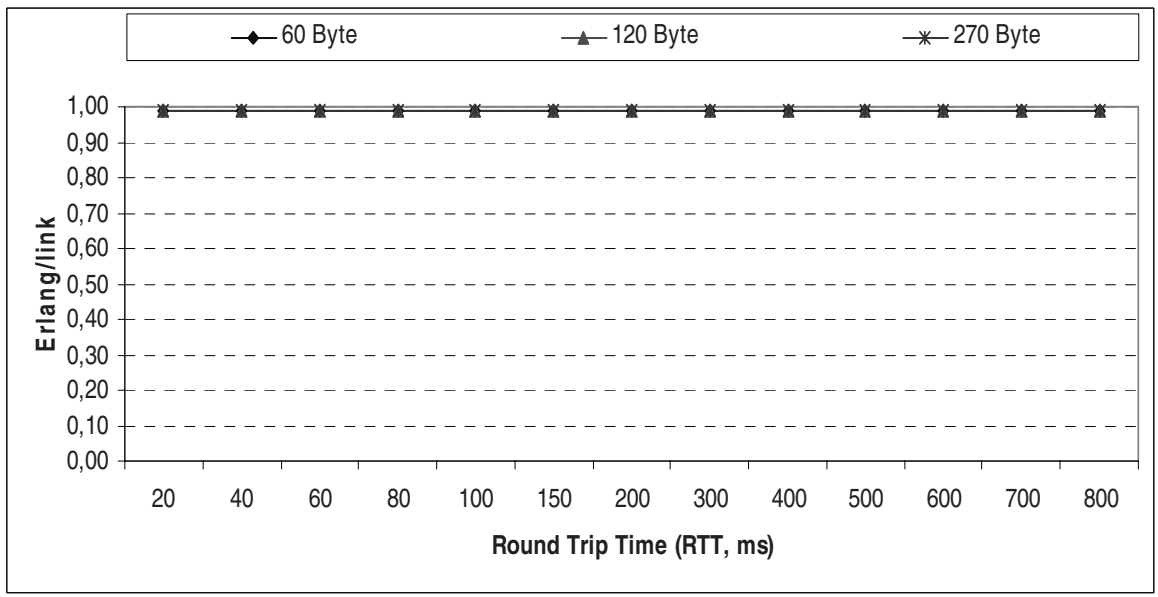

Fig. 8. SS7 Traffic Load vs. RTT (1 association, Rel. MB9-SAT)

\subsection{Bandwidth Availability Effects}

In order to evaluate satellite bandwidth availability effects, we observe system reactions increasing SS7 traffic, with PAMA Band set first to $2048 \mathrm{~Kb} / \mathrm{s}$ and then to 1024 
$\mathrm{Kb} / \mathrm{s}$; thank to Operative System change, there is no initial congestion, but two critical consequences appear (see Figure 9):

1) when Ethernet Band of generated traffic exceeds PAMA Band even only by $5 \%$, about the $50 \%$ of messages are lost (Used Band lower than Expected Band);

2) SS7 traffic has to be reduced to $50 \%$ of PAMA Band to clear congestion.

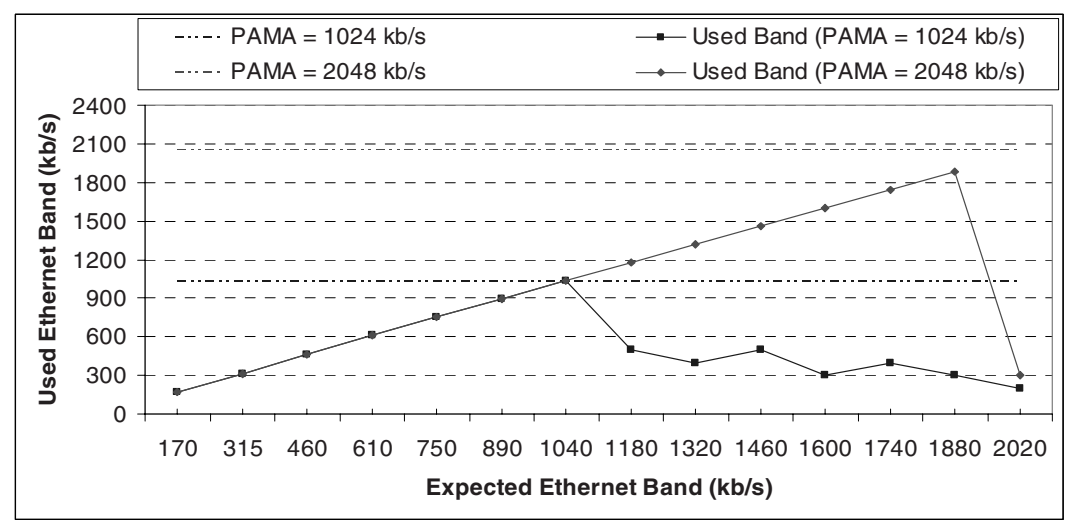

Fig. 9. Examples of Used Ethernet Band vs. Expected Ethernet Band

We can explain this behaviour by the following observations:

- the amount of acknowledge waiting data (so called OUTSTAND) grows with SS7 traffic band $\left(\mathrm{B}_{\mathrm{SS} 7}\right)$ and with the Acknowledge Time $\mathrm{T}_{\mathrm{ACK}}$, needed by SCTP to receive a transmitting data acknowledge and made up by Round Trip Time, possible DAMA Band Allocation Time $\left(\mathrm{T}_{\mathrm{ALL}}\right)$ and possible Queuing Time on SS7 receiving interface $\left(\mathrm{T}_{\mathrm{Q}}\right)$, according to:

$$
\text { OUTSTAND }=\mathrm{B}_{\mathrm{SS} 7} \cdot \mathrm{T}_{\mathrm{ACK}} \quad \mathrm{T}_{\mathrm{ACK}}=\mathrm{RTT}+\mathrm{T}_{\mathrm{ALL}}+\mathrm{T}_{\mathrm{Q}}{ }^{1}
$$

- OUTSTAND and CWND define respectively the traffic band injected in the network (named $\mathrm{B}_{\mathrm{IN}}$ ) and its maximum threshold (named $\mathrm{B}_{\mathrm{IN}, \max }$ ), beyond which Congestion Avoidance (CA) Algorithm takes action, according to:

$$
\begin{gathered}
\mathrm{B}_{\mathrm{IN}}=\text { OUTSTAND [Bytes] } 8 \text { [bit] } / \mathrm{T}_{\mathrm{ACK}} \\
\mathrm{B}_{\mathrm{IN}, \max }=\mathrm{CWND}[\text { Bytes }] \cdot 8[\mathrm{bit}] / \mathrm{T}_{\mathrm{ACK}}
\end{gathered}
$$

When $\mathrm{B}_{\mathrm{SS} 7}$ is constant and lower than PAMA Band, OUTSTAND is constant too, $\mathrm{T}_{\mathrm{ACK}}$ is just equal to RTT and $\mathrm{B}_{\mathrm{IN}}$ is equal to $\mathrm{B}_{\mathrm{SS} 7}$; apparently, this situation leads to set CWND at the highest value, so that Congestion Time $T_{C}$, needed by OUTSTAND to reach CWND, is as long as possible (at least longer than $\mathrm{T}_{\mathrm{ACK}}$ ) and the Satellite Band $\mathrm{B}_{\mathrm{S}}(\mathrm{PAMA}+\mathrm{DAMA})$ can be fully employed, without congestion limit.

$1 \mathrm{~T}_{\mathrm{Q}}$ will be omitted from now on, since it can be avoid by generating less than 1 Erlang/link on each of 30 Signalling Links per SG. 
But when $\mathrm{B}_{\mathrm{SS} 7}$ grows up of $\Delta \mathrm{B}$, exceeding PAMA Band before $\mathrm{B}_{\mathrm{IN} \text {, max }}$, the Satellite Allocation/De-Allocation (SAD) Algorithm causes a $T_{A L L}(1 \div 2 \mathrm{~s})$, which makes $\mathrm{T}_{\mathrm{ACK}}$ surely longer than $T_{C}$ : because of this, not only OUTSTAND (and thus $B_{\text {IN }}$ ) grows faster to CWND ( $\mathrm{B}_{\mathrm{IN}, \max }$ ) causing congestion, but also the higher CWND has been set, the heavier congestion goes, with both CWND and OUTSTAND falling down and oscillating, triggering SAD and CA Algorithms in cycle. The system returns to a stable condition only by traffic reduction to $50 \%$ of PAMA Band.

Facing such effects, increasing the SCTP associations' number appears no longer useful, as easy to check by using WAN Emulator first and then going back to satellite connection:

- without band limitation (WAN Emulator), setting Associations' Number $\mathrm{N}_{\mathrm{A}}=2$ makes accepted traffic double, RTT and CWND being equal, because accepted traffic is proportional to $\mathrm{N}_{\mathrm{A}}$ and CWND, inversely proportional to RTT;

- with band limitation (satellite connection), available band is however shared between $\mathrm{N}_{\mathrm{A}}=2$ associations, each of them going congested to half the traffic of previous case, since two associations with fixed CWND are equal to single association with doubled CWND, once again underlining its basic importance.

Therefore our final goal is to define the appropriate rules, which allow to correctly set CWND value, so that, when OUTSTAND reaches it, there both values stay still and any other traffic increase is rejected by periodic loss events, without performance collapse and simultaneously without wasting $\mathrm{B}_{\mathrm{s}}$.

\subsection{Defining an Operative Reference Model}

We can model the transmitting side of the system under test as shown in Figure 10, where the SS7 traffic band $\left(\mathrm{B}_{\mathrm{SS} 7}\right.$, generated by 30 Signalling Links, each at $0 . \mathrm{xx}$ Erlang rate) feeds OUTSTAND for the time $\mathrm{T}_{\mathrm{ACK}}$, being injected in the Fast Ethernet network as $\mathrm{B}_{\mathrm{ETH}}=\left(1+\alpha_{\mathrm{OH}}\right) \cdot \mathrm{B}_{\mathrm{IN}}$ (with $\alpha_{\mathrm{OH}}$ to calculate OverHead).

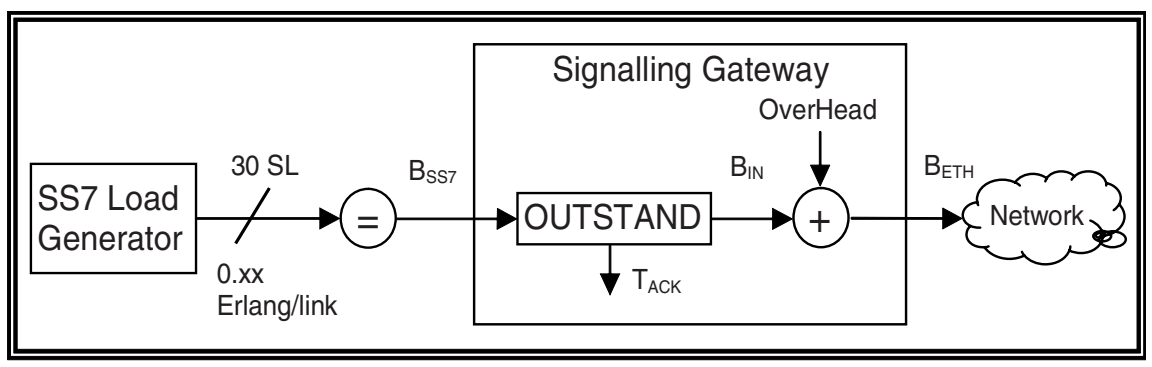

Fig. 10. System under Test transmitting scheme

Together with (1), (2), (3) and according to what expounded above, they let us formalize the following relations:

a) when CWND is such that $\left(1+\alpha_{\mathrm{OH}}\right) \cdot \mathrm{B}_{\mathrm{IN}, \max }$ is lower than PAMA Band and $\mathrm{B}_{\mathrm{SS} 7}$ increases of $\triangle \mathrm{B}$, OUTSTAND grows in function of $\Delta \mathrm{B}$ as

$$
\text { OUTSTAND }=\mathrm{B}_{\mathrm{SS7}} \cdot \mathrm{RTT}+\Delta \mathrm{B} \cdot \mathrm{RTT}
$$


and, if CWND is reached, CA Algorithm is able to keep them both to maximum value, rejecting any other traffic increase by periodic loss events, without performance collapse;

b) when CWND is such that $\left(1+\alpha_{\mathrm{OH}}\right) \cdot \mathrm{B}_{\mathrm{IN} \text {, max }}$ is greater than the whole $\mathrm{B}_{\mathrm{S}}$ and $\mathrm{B}_{\mathrm{SS} 7}$ increases of $\triangle \mathrm{B}$ over PAMA Band, OUTSTAND grows in function of $\Delta \mathrm{B}$ and of $\mathrm{T}_{\mathrm{ALL}}$ as

$$
\text { OUTSTAND }=\mathrm{B}_{\mathrm{SS} 7} \cdot \mathrm{RTT}+\Delta \mathrm{B} \cdot\left(\mathrm{RTT}+\mathrm{T}_{\mathrm{ALL}}\right)
$$

where the second term implies a transient dynamic rise, making OUTSTAND suddenly exceed CWND, so that CA Algorithm triggers the cycled action of SAD Algorithm, which drives SCTP association to a continuous congestion, cleared only by traffic reduction to $50 \%$ of PAMA Band;

c) when CWND is such that $\left(1+\alpha_{\mathrm{OH}}\right) \cdot \mathrm{B}_{\mathrm{IN} \text {, max }}$ is greater than PAMA Band, but lower than $\mathrm{B}_{\mathrm{s}}$, it's possible to overcome PAMA Band and to avoid congestion by keeping $\Delta \mathrm{B}$ under the opportune threshold.

All these conditions are schematically summed up in the three working cases of (6), whose knowledge allows a right configuration of transport parameters:

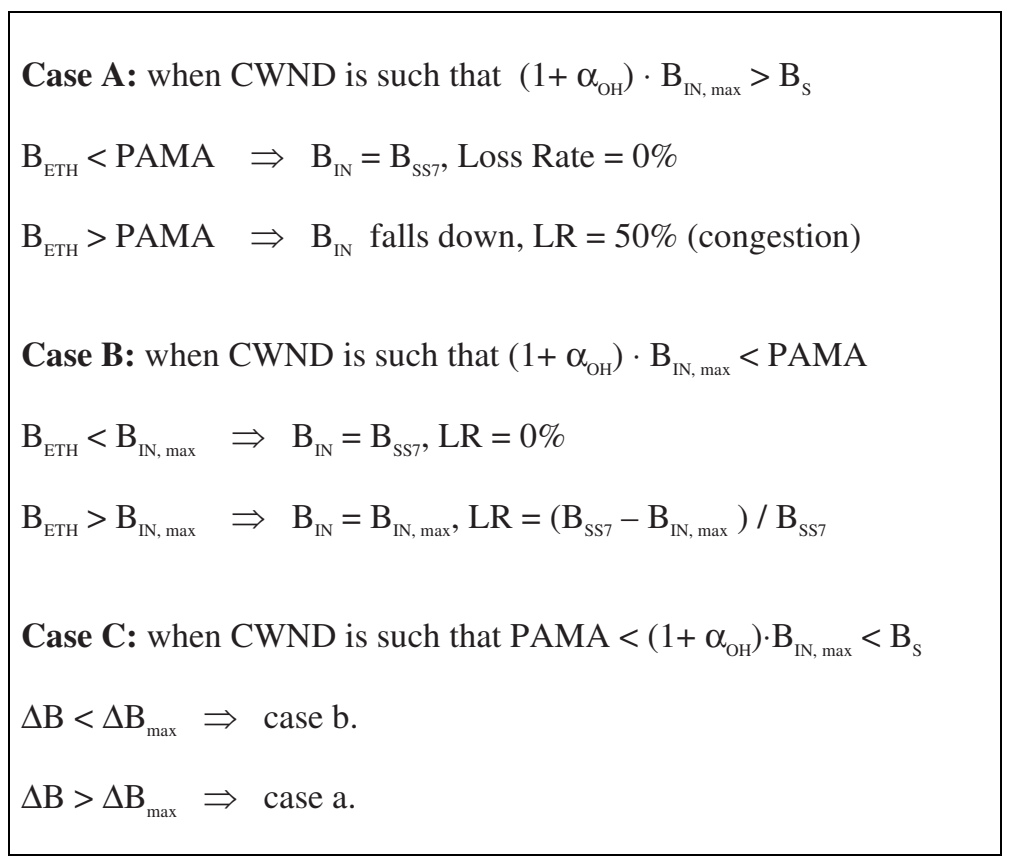

Finally, the study on $\mathrm{N}_{\mathrm{A}}$ number of SCTP associations can be summed up in the following:

$$
\mathrm{N}_{\mathrm{A}} \cdot \mathrm{CWND} \cdot 8 / \mathrm{T}_{\mathrm{ACK}}=\text { constant }=\mathrm{N}_{\mathrm{A}} \cdot \mathrm{B}_{\mathrm{IN}, \max }=\mathrm{B}_{\mathrm{IN}, \mathrm{MAX}}
$$

where $\mathrm{B}_{\mathrm{IN}, \mathrm{MAX}}$ is the total Maximum Injected Band, to be compared to $\mathrm{B}_{\mathrm{S}}$ according to (6), that means $\mathrm{B}_{\mathrm{IN}, \max }$ to be compared to $\mathrm{B}_{\mathrm{S}} / \mathrm{N}_{\mathrm{A}}$. 
From this viewpoint, also statements in paragraph 3.1 about Transmit Delays $\left(\mathrm{N}_{\mathrm{A}}=\right.$ 8) find justification:

- 8 SCTP associations with CWND maximum value 64000 are equivalent to a single association with CWND maximum value 516000, enough to reach PAMA Band and fall in congestion with Loss Rate $50 \%$ (case A);

- initial congestion dues to CWND too slow rise from initial value.

Expressions (1), (2), (3), (4) and (5) build up an operative reference model about the system under test.

\section{Test Methodology and Experimental Evidences}

According to previous achievements, it follows that:

1) system congestion find the trigger event in band availability, when PAMA Band is over and DAMA Band gets allocated in $\mathrm{T}_{\mathrm{ALL}}$;

2) two SCTP associations with fixed CWND are equivalent to one association with doubled CWND, on the basis of (5);

3) CWND is a significant parameter and shall be fixed on the basis of (6);

4) SS7 generated traffic increase shall take in consideration (6), as well.

To execute meaningful tests, system equipments are configured as follow:

- Satellite band: PAMA Band at both $2048 \mathrm{~Kb} / \mathrm{s}$ and $1024 \mathrm{~Kb} / \mathrm{s}$ with DAMA Band on demand till a total band of about $2150 \mathrm{~Kb} / \mathrm{s}$, configuration of SAD Algorithm in order to have the quickest allocation and the slowest de-allocation;

- Signalling Gateway: one SCTP association, with different CWND initial value.

From the whole tests we find that there isn't any dependence on SS7 routing (DPC or GTT) and different MSU length means essentially different OverHead fraction $\alpha_{\mathrm{OH}}$ (inversely proportional to MSU length), SS7 generated traffic being equal.

\subsection{Experimental Evidences}

Starting with the suggested value for CWND (384000 Bytes), $\mathrm{B}_{\mathrm{IN} \text { max }}$ is about 4600 $\mathrm{Kb} / \mathrm{s}$ (considering $\mathrm{T}_{\mathrm{ACK}}=\mathrm{RTT}=650 \mathrm{~ms}$ ) and, whatever OverHead fraction we use, the system is in Case A for both PAMA Band choices. With 270 Bytes long MSUs, $\alpha_{\mathrm{OH}}$ is about 0.16 and $\Delta \mathrm{B} \approx 120 \mathrm{~Kb} / \mathrm{s}$ corresponds to steps of about $140 \mathrm{~Kb} / \mathrm{s}$ in Ethernet Band. Tests for both PAMA values show that no messages are lost until PAMA Band is quite over; then, Ethernet Band falls down and start to oscillate, the system goes under congestion with Loss Rate of about $50 \%$ and turns stable only after $\mathrm{B}_{\mathrm{IN}}$ is hardly reduced (as yet shown in Figure 9).

Changing CWND value to 154000 Bytes, when using 270 Bytes long MSUs, the system is in Case B with PAMA Band set to $2048 \mathrm{~Kb} / \mathrm{s}$ and a null value for DAMA Band, because RTT rises from $650 \mathrm{~ms}$ to $750 \mathrm{~ms}$ while approaching PAMA, so that $\mathrm{B}_{\mathrm{IN}, \max } \approx 1650 \mathrm{~Kb} / \mathrm{s}$ and $\left(1+\alpha_{\mathrm{OH}}\right) \cdot \mathrm{B}_{\mathrm{IN}, \max } \approx 1910 \mathrm{~Kb} / \mathrm{s}$.

Tests show that $\mathrm{B}_{\mathrm{IN}}$ rises till $\mathrm{B}_{\mathrm{IN} \text {, max }}$ and then any traffic increase is periodically rejected, without compromising system performances (see Figure 11). 
If DAMA Band is set to $250 \mathrm{~Kb} / \mathrm{s}$, RTT is about $650 \mathrm{~ms}$ also while approaching PAMA Band, so that $\mathrm{B}_{\mathrm{IN}, \max } \approx 1850 \mathrm{~Kb} / \mathrm{s}$ and $\left(1+\alpha_{\mathrm{OH}}\right) \cdot \mathrm{B}_{\mathrm{IN}, \max } \approx 2150 \mathrm{~Kb} / \mathrm{s}$ : the system is lightly in Case $C$.

A test with 270 Bytes long MSUs shows that, with $\Delta \mathrm{B} \approx 130 \mathrm{~Kb} / \mathrm{s}$ (it corresponds to steps of about $150 \mathrm{~Kb} / \mathrm{s}$ in Ethernet Band), congestion comes only when $\mathrm{B}_{\mathrm{IN}, \max }$ exceeds PAMA Band, being kept at about $2100 \mathrm{~Kb} / \mathrm{s}$ by periodic loss.

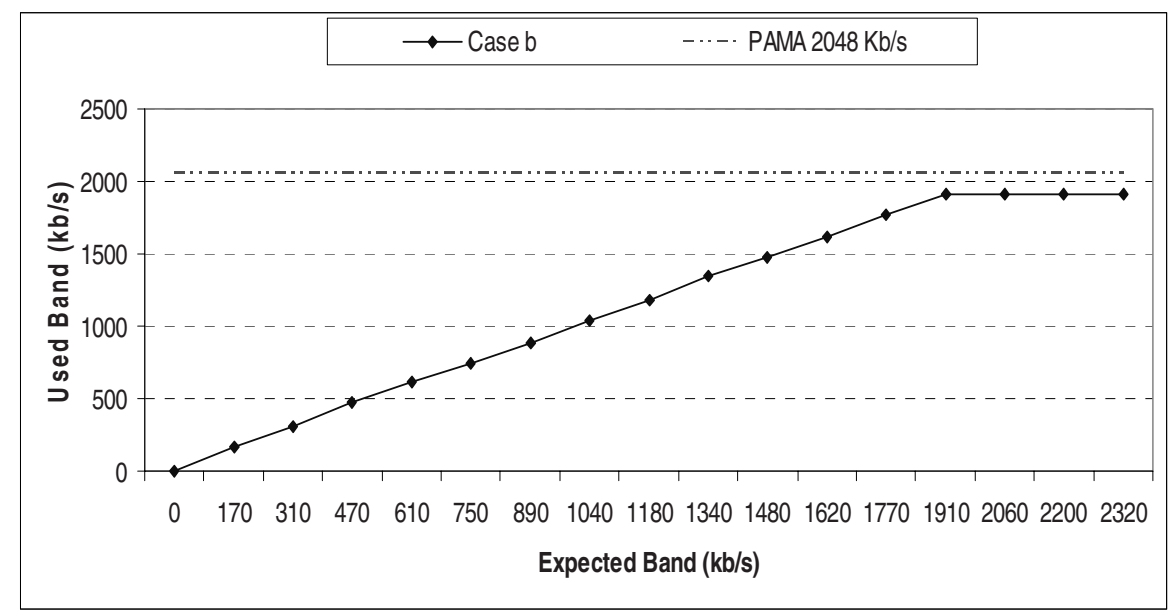

Fig. 11. Examples of tests results in case B

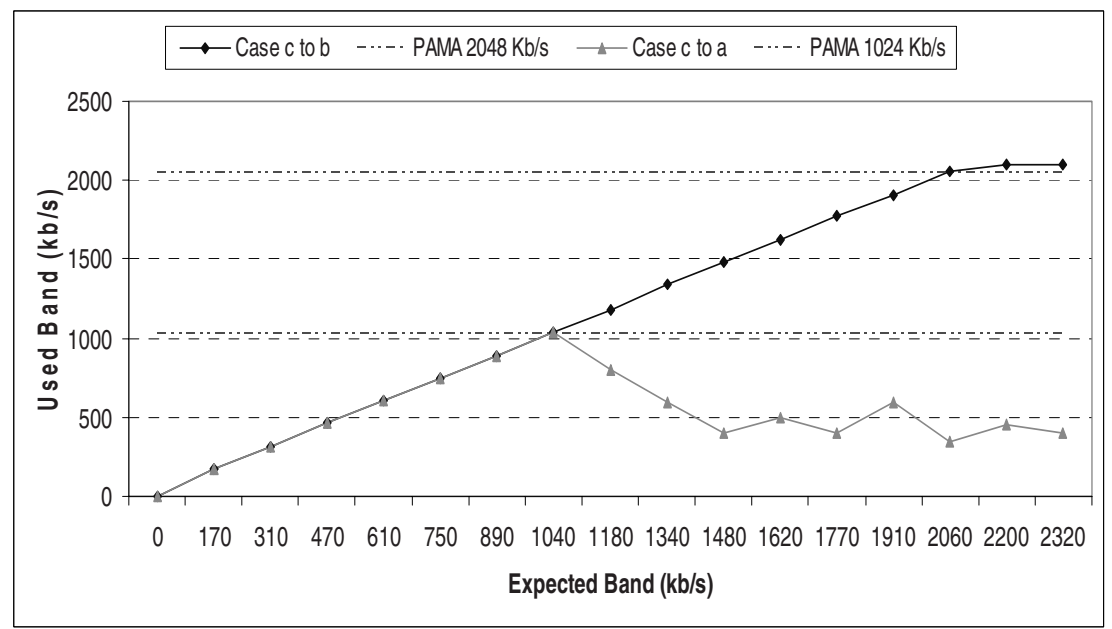

Fig. 12. Examples of tests results in case $C$

Results with 60 Bytes long MSUs are quite the same: RTT grows to $750 \mathrm{~ms}$ and $\left(1+\alpha_{\mathrm{OH}}\right) \cdot \mathrm{B}_{\mathrm{IN}, \max } \approx 2300 \mathrm{~Kb} / \mathrm{s}$, the same $\Delta \mathrm{B}$ is accepted and $\mathrm{B}_{\mathrm{IN}, \max }$ stay around 2100 $\mathrm{Kb} / \mathrm{s}$ by periodic loss. 
The situation is stressed by setting PAMA Band to $1024 \mathrm{~Kb} / \mathrm{s}$, where $\Delta \mathrm{B} \approx 190 \mathrm{~Kb} / \mathrm{s}$ keeps the system in Case $B$, while $\Delta \mathrm{B} \approx 250 \mathrm{~Kb} / \mathrm{s}$ drives it to Case $A$ (see Figure 12).

\subsection{Open Issues}

Since the system behavior appears quite complicated, there are still some details to be clarified, that our future experimental activity will aim to.

The main aspects to be investigated are:

- $\mathrm{T}_{\mathrm{ALL}}$ dynamic contribution to OUTSTAND quick growth;

- $\Delta \mathrm{B}_{\max }$ correlation with SCTP parameters, as CWND, and with the system's parameters, as $\mathrm{T}_{\mathrm{ACK}}, \mathrm{T}_{\mathrm{ALL}}$, etc.;

- Transit Delays of SS7 messages during DAMA Band allocation may exceed specifications' time-outs.

\section{Conclusion}

Our trial activity on SoIP over Satellite connection shows that it can be used in operations choosing in the right way the mentioned parameters. It has been underlined how its critical aspects (Transmit Delay and Bandwidth Availability) can influence the whole system's performances. While delay consequences (RTT 640-680 ms) seems to be overcome by configuring the maximum size of the SCTP Congestion Window at higher value (or equivalently by using more than one SCTP association), the fact that satellite bandwidth can be provided both as permanent (PAMA) and dynamic (DAMA) appears not to be solved at all by these actions, because the Satellite Allocation/De-allocation Algorithm can interact with SCTP Congestion Avoidance Algorithm in a dangerous way for system stability, if parameters aren't configured with special attention.

In order to guide configuration choices we propose an operative reference model, whose usefulness has been confirmed by testing validation, even if still more study is needed to have it completed.

\section{References}

[1] R. Stewart et al. "SCTP: Stream Control Transmission Protocol", IETF RFC 2960, October 2000.

[2] K. Morneault et al., "SS7 MTP2-User Adaptation Layer (M2UA) - draft-ietf-sigtranm2ua-15 work in progress", February 2002.

[3] G. Sidebottom et al., "SS7 MTP3-User Adaptation Layer (M3UA) - draft-ietf-sigtranm3ua-12 work in progress", February 2002.

[4] J. Loughney et al., "SS7 SCCP-User Adaptation Layer (SUA) - draft-ietf-sigtran-sua-12 work in progress", February 2002.

[5] T. George et al., "SS7 MTP2-User Peer-to-Peer Adaptation Layer (M2PA) - draft-ietfsigtran-m2pa-04 work in progress", February 2002.

[6] G. Maggiore , F. Piovan et al., "VoIP and SoIP testing methodology, TIM experience", SCI2002 Orlando, July 2002 
[7] ITU-T Recommendation Q.543, Digital Exchange Performance Design Objectives, ITUT, March 1993.

[8] ITU-T Recommendation Q.706, Signalling System N.7 - Message Transfer Part Signalling Performance, ITU-T, March 1993

[9] ITU-T Recommendation Q.716, Signalling System N.7 - Signalling Connection Control Part (SCCP) Performance, ITU-T, March 1993 